\title{
Les noms d'organisations politiques imaginaires : analyse d'un générateur automatique de noms de groupuscules
}

About the names of imaginary political organizations: analysis of an automatic generator of small group names

Los nombres propios de organizaciónes políticas imaginarias: análisis de un generador automático de nombres de grupúsculos

\section{Alice Krieg-Planque}

\section{OpenEdition \\ Journals}

Édition électronique

URL : https://journals.openedition.org/mots/25132

DOI : $10.4000 /$ mots. 25132

ISSN : $1960-6001$

Éditeur

ENS Éditions

Édition imprimée

Date de publication : 11 juillet 2019

Pagination : 15-37

ISBN : 979-10-362-0170-7

ISSN : 0243-6450

\section{Référence électronique}




\section{Les noms d'organisations politiques imaginaires : analyse d'un générateur automatique de noms de groupuscules}

Ce texte met en évidence certains aspects des représentations langagières associées aux noms propres d'organisations politiques, à travers l'analyse d'un document publié sur une page web consacrée à des contenus politiques humoristiques : la page Mèmes Royalistes. De façon inhabituelle par rapport à une étude plus classique des noms de partis (histoire du choix du nom, analyse sémantique des dénominations, étude des siglaisons, etc.), nous nous appuyons ici avant tout sur des données issues de mondes fictifs ou imaginaires (romans, séries télévisées, créations ludiques, etc.). Dans le cas présent, le document analysé est un générateur automatique de noms de groupuscules, conçu par Mèmes Royalistes, et qui a fait l'objet d'une large diffusion sur les réseaux sociaux numériques. À travers l'étude de ce corpus quelque peu atypique, il s'agit de montrer comment l'étude d'un certain type de nom d'organisation politique imaginaire enrichit la compréhension des dénominations de groupes humains constitués sur une base politique. Nous partons de l'idée que l'expression de représentations langagières - ici, observable dans le générateur édité par Mèmes Royalistes - participe de jugements sur la vie politique française : là comme dans bien d'autres domaines, la mise en scène d'un monde «imaginaire » éclaire la façon dont le monde «réel » est appréhendé. Jusqu'à un certain point, cette mise en scène contribue à produire ce réel, à travers des effets de sens que nous essaierons de décrire et d'interpréter.

Dans une première partie, nous présentons le document étudié et le cadre d'analyse que nous proposons. Il s'agit non seulement, bien sûr, de situer Mèmes Royalistes et sa place dans l'expression de l'humour politique en ligne, mais aussi de souligner que l'onomastique partisane gagne à s'intéresser aux noms propres d'organisations politiques qui apparaissent dans des mondes fictifs. Dans une deuxième partie, nous montrons combien le document analysé soutient la vision doctrinale de son auteur. Aux effets rhétoriques de disqualification propres aux générateurs automatiques d’énoncés s’ajoutent ici 
la représentation d'une société atomisée dans une France divisée, et un travail lexical complexe qui, tout en présentant les mots comme des «marqueurs» politiques, semble les vider de leur sens. L'ensemble de cette activité métadiscursive, qui joue sur la combinatoire de la langue, sur les structures syntaxiques et sur le lexique disponible, fait apparaître la doctrine royaliste maurrassienne comme un courant qui permettrait de redonner du sens à la politique et de son unité à la nation française. L'ensemble de notre contribution s'attache à illustrer combien l'analyse du discours doit toujours être menée en contexte et en situation.

\title{
«Toi aussi invente ton groupuscule d'extrême-gauche / d'extrême-droite " : un document et son cadre d'analyse
}

\author{
Les «mèmes internet » et l'expression de l'humour politique \\ en ligne : la page Mèmes Royalistes
}

Le document étudié ici est composé de deux visuels, intitulés respectivement «Toi aussi invente ton groupuscule d'extrême-gauche» et «Toi aussi invente ton groupuscule d'extrême-droite» (voir annexes 1 et 1 bis). Ces visuels ont été réalisés par le collectif Mèmes Royalistes, qui les a mis en ligne fin 2017 sur ses comptes Facebook et Twitter.

Mèmes Royalistes se présente lui-même sur sa page Facebook comme « un collectif informel de graphistes » aux objectifs suivants :

Nous nous inscrivons dans cette démarche de «complot à ciel ouvert» développé par Charles Maurras dans Si le coup de force est possible. Nous déconstruisons l'état d'esprit républicain de façon humoristique et notre objectif ultime est évidemment d'instaurer une monarchie traditionnelle, héréditaire, décentralisée et antiparlementaire. Toutefois, nous ne nous faisons pas d'illusions sur la portée de cette page qui cherche avant tout à faire rire et à susciter la curiosité. ${ }^{1}$

La page Facebook de Mèmes Royalistes a été lancée le 9 septembre 2017, et constitue la principale tribune du collectif. Elle est relayée par le compte Twitter du même nom, créé peu après. Devant le succès remporté par sa page Facebook, Mèmes Royalistes a lancé en juin 2018 un magazine titré Création (avec un effet graphique qui suggère l'anagramme Réaction) : destiné à un public jeune, le magazine présente à la fois des articles politiques de fond et des contenus satiriques. Le premier numéro, disponible en ligne gratuitement ${ }^{2}$ et

1. Page Facebook de Mèmes Royalistes, rubrique «Qui sommes-nous? », texte daté du 5 juin 2018, https://www.facebook.com/MemesRoyalistes/ (consulté le 03/04/2019).

2. Version feuilletable en ligne : https://fr.calameo.com/read/00561409557c876b2461a (consulté le 03/04/2019). Version téléchargeable au format pdf : http://www.mediafire.com/ file/edzzgnslzdtkwda/Magazine+Création.pdf (consulté le 03/04/2019). 
diffusé en version imprimée dans des contextes militants (par exemple à l'université d'été de l’Action française, en août 2018), était consacré à la personnalité de Charles Maurras et à sa doctrine du «nationalisme intégral», dont le magazine explique et défend les idées.

Même si son succès d'audience sur Facebook et sur Twitter est notable, le collectif Mèmes Royalistes n'est qu'un élément, parmi d'autres, de la présence des mouvances royalistes sur internet, dont le journaliste Bruno Lus a souligné la vivacité3. Au-delà des mouvances royalistes, c'est l'extrême droite dans son ensemble et dans sa diversité qui a très tôt investi les possibilités offertes par internet, depuis la création précoce d'un site web par le Front national au milieu des années 1990 (Dézé, 2011) jusqu'aux usages contemporains des sites d'information, forums, réseaux sociaux et plateformes de partage de vidéos, constituant ce qui a pu être appelé la «fachosphère » (Albertini, Doucet, 2016).

Mais le collectif Mèmes Royalistes ne témoigne pas seulement du fort activisme des extrêmes droites sur internet. Plus encore peut-être, il illustre la complexité sémiotique et discursive de l'humour politique en ligne. Celui-ci, qui est le fait d'acteurs politiques et idéologiques variés, réalise des formes riches et parfois subtiles de parodie, de pastiche, de satire et de détournement. Le web participatif enrichit encore ces formes grâce à différents types de réaction (like, commentaire, etc.) et de propagation (retweet, partage, etc.). Certaines formes d'humour relèvent d'expressions relativement classiques, quand d'autres apparaissent plus nettement comme des genres et des signes «natifs du web», tels que le recours aux « hashtags», les " gifs», les « lolcats», ou encore les «mèmes».

Les «mèmes», ou «mèmes internet», sont un genre de document (voir Gautier, Siouffi éd., 2016 ; Clément, Fernandez, Puel, 2016) que l'on peut définir sommairement comme un visuel de faible qualité graphique apparente (mauvaise pixellisation, dysharmonie des couleurs ou des polices de caractère, etc.) destiné à la dissémination en ligne ("viralité»), et caractérisé par une dimension humoristique, par des formes d'intertextualité (allusions, citations, etc.) et par la juxtaposition atypique d'objets visuels sans corrélations apparentes (effet de photomontage), le tout produisant en général un aspect un peu potache et sarcastique. L'utilisateur peut produire des « mèmes » grâce à des sites spécialisés en création de mèmes (Quickmeme.com, MemeCreator.org, etc.). Le mème est rapidement devenu le mode d'expression exclusif de certaines pages Facebook dédiées à l'humour politique ${ }^{4}$, compte tenu de sa rapidité de lecture (une image et quelques mots, ou une courte vidéo), de la

3. Bruno Lus, «Youtubeurs, "mèmes” et Tweet cinglants... la féroce bataille des royalistes sur Internet », Le Monde, 21 février 2018.

4. Quelques exemples sur Facebook: Mème de propagande pour une zad impérialiste, Mèmes Anarchistes pour que les mauvais jours finissent, Politise un film, LaFrancelnsoumeme, BlagueBlock, etc. 
connivence qu'il permet d'établir au sein de communautés de valeurs (pouvoir fédérateur), des détournements et défigements qu'il encourage (appropriations par les utilisateurs), et de sa portée sarcastique ou drolatique. Les deux visuels étudiés dans cet article ne sont pas très caractéristiques du mème comme genre de document. Mais ils s'y rattachent par leur environnement éditorial : de fait, le compte Mèmes Royalistes diffuse presque exclusivement des mèmes.

Comme beaucoup de formes de l'humour politique en ligne, les mèmes demandent souvent à la fois une culture du web (connaissance de signes et de genres spécifiques du web, de codes sémiotiques particuliers, actualité des réseaux, etc.), et une culture politique (connaissance parfois fine des idées politiques et de leur histoire, des positionnements, du vocabulaire, des arrière-plans idéologiques, etc.). Les messages publiés par Mèmes Royalistes n'échappent pas à cette exigence de lecture et d'interprétation, qui est aussi par ailleurs une ressource pour la diffusion des idées. En effet, les messages humoristiques ou parodiques font l'objet, sur internet, d'une circulation complexe, au cours de laquelle la prise en charge énonciative peut connaître des réorientations étonnantes. On se souvient ainsi de Christine Boutin se ridiculisant sur un plateau de télévision en citant très sérieusement un article du site parodique Le Gorafi, croyant qu'il s'agissait là de véritables propos gouvernementaux 5 . Dans une perspective propagandiste et à des fins de déstabilisation, il arrive aux sites de «réinformation » d'extrême droite (Novopress, Fdesouche, Boulevard Voltaire, etc.) de rediffuser des informations du Gorafi ou de Nordpresse tout en sachant qu'il s'agit là de messages parodiques, de manière à créer de la confusion. Les messages humoristiques en ligne sont ainsi pris dans des jeux complexes de circulation, de prise en charge et de reprises, qui peuvent en faire, en dépit parfois d'une légèreté apparente, des vecteurs de combat culturel dans un cadre idéologique. C'est aussi ce que vise Mèmes Royalistes qui, selon ses propres termes, en «propos[ant] un contenu humoristique politisé à travers les mèmes ${ }^{6}$, cherche à faire connaître et propager les options du nationalisme intégral prôné par Charles Maurras. Car, comme le rappelle Création, pour que le «coup de force » qui renversera la République soit possible, « un long travail de propagande doit permettre de convertir autant les masses que les élites civiles et militaires aux idées royalistes »7.

Ces conditions de production guident notre analyse : "Toi aussi invente ton groupuscule d'extrême-gauche» et «Toi aussi invente ton groupuscule d'extrême-droite » ne sauraient être détachés de l'énonciation qui les fait advenir et leur donne sens.

5. Christine Boutin, présidente d’honneur du Parti chrétien-démocrate, invitée sur le plateau de BFM TV, lundi 3 février 2014 .

6. Page Facebook de Mèmes Royalistes, rubrique «Qui sommes-nous ? », texte daté du 5 juin 2018.

7. «La prise du pouvoir chez Maurras », Création, nº 1, juin 2018, p. 16. 
Les noms propres d'organisations politiques imaginaires : nommer le collectif politique dans des mondes fictifs

Les dénominations qui nous intéressent dans le document de Mèmes Royalistes sont celles que le générateur permet de créer: CollectifAntifasciste International, Front Totalitaire Français, Action Autonome Révolutionnaire, Division Populaire Nationaliste, etc. De telles dénominations relèvent de la catégorie des noms propres d'organisations politiques imaginaires, catégorie qu'il convient de définir.

Par «nom propre d'organisation politique imaginaire», nous entendons un nom propre affecté à un groupe humain qui est constitué sur une base politique et qui appartient à un monde imaginaire. En tant que groupes humains constitués sur une base politique, les référents concernés sont donc susceptibles d'être désignés par des noms collectifs (Lecolle, 2014) tels que cercle, clan, clique, faction, fédération, groupuscule, ligue, parti, union, etc. (mais non pas par exemple orchestre, chœur, chambrée, etc., dont la base politique n'est pas manifeste). Le groupe désigné est imaginaire en ce sens qu'il apparaît dans un monde dont les réalités sont uniquement langagières et symboliques (textes de fiction, films, etc.).

La définition que nous venons d'indiquer pourrait laisser penser que les réalités discursives concernées sont rares. Il n'en est rien. Nombreux sont les romans, films, séries, jeux vidéo et autres expressions artistiques en tous genres qui offrent des exemples de noms propres d'organisations politiques imaginaires.

Les œuvres dystopiques sont bien entendu un espace privilégié pour ce type de dénomination. On pense bien sûr à l'ANGSOC, nom du parti unique dans le roman 1984 de George Orwell, ou à Globalia, nom de l'État mondial dans le roman éponyme publié par Jean-Christophe Rufin. On peut penser également à Norsefire (nom du parti fasciste dans le film V pour Vendetta), à La Troisième Vague (nom du mouvement fascisant dans le film et le roman La Vague), ou à La Hanse, La Ligne Rouge, Le Quatrième Reich (noms de confédérations de stations dans l'œuvre post-apocalyptique Métro 2033). On rencontre également des noms propres d'organisations politiques imaginaires dans des œuvres relevant plutôt de l'horreur (communautés et groupes de la série télévisée The Walking Dead), de l'univers des comics (clubs et confréries des X-Men), ou des œuvres plus classiques de science-fiction (partis politiques du Cycle de Fondation d'Isaac Asimov).

De manière satirique, la bande dessinée Monsieur le Ministre de Binet (1989) relate l'histoire de Lucien Grangarçon, personnalité insipide dont la trajectoire tout autant que l'exercice du pouvoir sont tournés en dérision, mis en situation dans un monde politique et médiatique marqué par la médiocrité. Le parti politique au sein duquel Lucien Grangarçon fait carrière s'appelle l'UDDD 
(Union Démocratique des Démocrates pour la Démocratie), offrant un exemple de nom propre d'organisation politique imaginaire à caractère parodique (voir annexe 3).

Sur internet, certaines pages consacrées à la diffusion de mèmes politiques portent parfois des noms propres d'organisations politiques imaginaires. C'est le cas par exemple de comptes Facebook intitulés LaFrancelnsoumeme, Courant Anarchostalinien, Commune insurrectionnelle de Versailles, ou encore Front Prolétarien Mao-Guevariste Hoxaiste de Sciences Po. Les noms propres d'organisations politiques imaginaires concernent ainsi aisément les supports d'autopublication numériques. La liste pourrait être prolongée. Le chantier ne manque pas de données empiriques pour qui voudrait y œuvrer.

\section{L'analyse du nom propre d'organisation politique : enrichir les corpus étudiés grâce aux noms imaginaires}

L'étude des noms de partis politiques (et plus largement de syndicats, de listes électorales, de coalitions, etc.), dont le présent numéro de Mots dresse un bilan et pour laquelle il propose des perspectives, gagnerait à enrichir ses corpus avec des noms propres d'organisations politiques imaginaires, dont nous venons de souligner l'existence. De même que l'intérêt porté par la linguistique aux «langues imaginaires» et aux «langues construites» a pu nourrir l'étude des langues et du langage (Yaguello, 2006), un intérêt pour les noms propres imaginaires enrichirait certainement l'onomastique partisane.

De fait, les noms propres d'organisations politiques imaginaires partagent avec les noms d'organisations « réelles» les caractéristiques les plus habituellement reconnues au nom propre d'organisation politique :

- le nom est choisi par ceux qu'il va dénommer collectivement, et non pas par un acteur extérieur; ceci lui confère une dimension revendicative (le détenteur du nom prétend à quelque chose à travers son nom, ce qui n'est pas le cas quand le nom est donné de l'extérieur); mais ceci fait par ailleurs porter sur le détenteur du nom une certaine responsabilité (il doit «assumer » le nom qu'il s'est donné à lui-même);

- le nom est formé de mots qui appartiennent au lexique courant et qui sont porteurs de sens, ce qui confère au nom propre d'organisation politique un certain degré de «transparence descriptive vis-à-vis de [son] référent», pour reprendre l'analyse de Michelle Lecolle (2014, p. 2265) à propos de certaines dénominations de groupes sociaux;

- le nom est à mettre en relation avec un «acte de baptême» et il participe d'usages performatifs : le fait de nommer l'organisation lui donne une existence, sous réserve que soient remplies diverses conditions sociales qui garantissent la réussite de la dénomination comme institution (procédures, inscriptions sur des registres, etc.). 
Les noms propres d'organisations politiques imaginaires ressemblent ainsi singulièrement à des noms propres d'organisations politiques « réelles ». Pourtant, et précisément parce que leurs enjeux sont ceux de mondes fictifs, les noms propres d'organisations politiques imaginaires peuvent se permettre des libertés et des fantaisies que ne peuvent pas s'autoriser leurs analogues dans la «vie réelle».

La parodie, la caricature, la dérision, l'humour, et autres procédés créatifs, rendent possibles l'exagération, l'excès, l'accentuation des traits, et plus globalement une intelligibilité renouvelée de la réalité. Pour le chercheur, cette autre vision de la réalité - présente dans les romans, films, jeux et expressions humoristiques que nous avons évoqués - donne accès à des représentations langagières, à des conceptions de la nomination, à des appréciations sur les usages des noms, etc. Dans cet article, s'agissant de noms propres d'organisations politiques, ce sont, à travers ces points de vue, des jugements sur la vie politique et sur des fonctionnements institutionnels ou sociaux qui peuvent s'exprimer.

Dans ses différents écrits sur la langue anglaise, George Orwell dénonçait les troncations et les siglaisons, dans lesquelles il voyait un symptôme à la fois de la technicisation déshumanisante de la société et de l'euphémisation typique, selon lui, des discours politiques. Dans le roman 1984, le nom qu'Orwell donne au parti totalitaire au pouvoir a précisément recours à ces procédés décriés par l'écrivain britannique : INGSOC, pour «English Socialism» (ce que les traductrices en français ont traduit par $A N G S O C$, puis plus récemment par SOCIANG, pour "Socialisme Anglais»).

La bande dessinée de Binet évoquée plus haut, Monsieur le Ministre, est une satire de la vie politique française. L'auteur y dénonce la flagornerie de journalistes paresseux et blasés, la vanité et la suffisance des responsables politiques, l'importance du protocole et des attributs du prestige, lesquels semblent faits principalement pour dissimuler la médiocrité des élites au pouvoir. Binet dénonce aussi les appareils politiques aux contenus idéologiques vides, mais qui offrent des carrières aux élus et aux héritiers, et différents avantages aux entourages et aux courtisans. Comment ne pas voir dans ce nom d'UDDD (Union Démocratique des Démocrates pour la Démocratie) une expression du sentiment des usages de la langue en rapport direct avec cette vision de la politique?

Les noms propres d'organisations politiques imaginaires apparaissent ainsi comme un objet pertinent pour observer l'expression de représentations langagières, y compris en tant que celles-ci relèvent d'appréciations sur la vie politique. C'est ce que nous proposons d'approfondir à présent au sujet du générateur automatique de noms de groupuscules réalisé par Mèmes Royalistes. 


\section{L'expression des représentations langagières au service d'un jugement sur la vie politique française}

En prenant pour exemple le document élaboré par Mèmes Royalistes (voir annexes 1 et 1 bis), nous montrons à présent en quoi l'expression de représentations langagières à propos du nom propre d'organisation politique éclaire la vision portée par ce collectif royaliste : il s'agit d'analyser ce que les amplifications et les inventions permises par l'humour nous disent des représentations associées aux noms d'organisations politiques, et, partant, ce qu'elles nous disent de jugements portés sur la vie politique et sociale. En effet, à travers ce document, Mèmes Royalistes indique un certain point de vue sur la façon de déterminer les noms propres, sur la structure syntaxique de ces noms, et sur le lexique utilisé pour leur formation. Nous examinons successivement ces trois points.

\section{S'exprimer au moyen d'un générateur automatique d'énoncés :} des effets rhétoriques de disqualification

Dans le document étudié, une certaine négativité provient du nom collectif humain groupuscule, dont M. Lecolle (2016) a relevé le potentiel axiologique négatif, associé notamment à une certaine dangerosité et/ou une certaine violence (comparé par exemple à mouvement). Mais la négativité sur laquelle nous voulons insister ici est celle que produit le procédé choisi par Mèmes Royalistes pour évoquer la manière dont sont dénommés ces groupuscules, à savoir un générateur automatique d'énoncés, dont nous mettons en évidence les effets rhétoriques de disqualification.

Procédé de formation des discours bien connu de l'écriture à contrainte pour ses effets poétiques et/ou comiques (cadavre exquis du surréalisme, Cent mille milliards de poèmes de Queneau, etc.), la génération automatique de phrases et de textes est également souvent mobilisée dans des perspectives de critique des discours politiques et institutionnels. Elle sert à dénoncer les propos convenus, la phraséologie, les modèles de texte prêts à l'emploi (lettres de candidature, discours de vœux, etc.), la «novlangue» ou la «langue de bois» (Krieg-Planque, 2018). De fait, les générateurs automatiques d'énoncés s'attaquent au caractère préfabriqué de certains discours, dont ils rendent observable de manière amusante et concrète la dimension prévisible, ce par quoi ils en subvertissent l'autorité (Krieg-Planque, 2015). Favorisés par les développements de l'informatique, les générateurs d'énoncés sont très prisés des sites web humoristiques, y compris des sites de mèmes. C'est ce qu'illustre le document diffusé par le compte Mèmes Islamogauchistes, qui tourne en dérision les arguments de la présidence Macron en soulignant leur caractère tout à la fois vide et stéréotypé (voir annexe 4). 
Dans «Toi aussi invente ton groupuscule d'extrême-gauche» et «Toi aussi invente ton groupuscule d'extrême-droite», ce mode d'élaboration qu'est le générateur est particulièrement riche d'effets de sens.

Une première observation concerne l'entité responsable de la dénomination. En effet, qui détermine le nom du groupuscule ? À la différence du cas de figure dans lequel c'est un collectif qui détermine son propre nom, c'est ici un individu seul («toi aussi invente ton groupuscule») qui procède au choix du nom propre. Le document donne ainsi à voir une représentation d'utilisateurs isolés, œuvrant en quelque sorte à leur propre «acte de baptême ». Le déterminant possessif ( «invente ton » et non pas « invente un») accentue cette dimension individualiste. On peut voir ici, en cohérence avec le nationalisme intégral de Maurras, une dénonciation - certes ironique et indirecte - du caractère atomisé de la société française et de l'individualisme des démocraties libérales, que rejette précisément la pensée maurrassienne. Nous reviendrons plus loin sur cette dénonciation.

Une deuxième observation concerne les critères sur la base desquels l'utilisateur détermine le nom du groupuscule. Le ressort humoristique du document - mais qui n'est pas dénué d'effets de sens - est bien évidemment que ces critères sont contingents, liés à des propriétés fortuites de l'individu créateur du nom (le signe du zodiaque ou la taille de la personne auraient aussi bien pu être choisis). Au contraire de ce que seraient des propriétés signifiantes (confession religieuse, idéaux politiques, goûts culturels, etc.), les propriétés qui déterminent ici la dénomination sont dénuées de signification. Un premier élément d'arbitraire et de «non-sens » apparaît ici, qui est renforcé par l'observation qui suit, plus spécifique au dispositif mobilisé.

Notre troisième observation porte sur la nature même de ce dispositif qu'est un générateur, et sur ce qu'il induit. La caractéristique inhérente des générateurs automatiques d'énoncés est qu'ils livrent au hasard la présence des unités appartenant à un même paradigme, laissant du même coup apparaître ces unités comme substituables les unes aux autres (Krieg-Planque, 2015). Ordinairement, dans la vie « réelle », les noms propres d'organisations (partis politiques, mais aussi entreprises, collectivités territoriales, etc.) sont le fruit d'une activité métalinguistique approfondie, raisonnée, parfois même professionnelle et rémunérée. Ici, bien au contraire, ces noms sont composés de manière aléatoire. En donnant les mots à voir comme substituables les uns aux autres, les générateurs les présentent comme vides de sens : si un mot peut être indifféremment remplacé par un autre, c'est bien qu'il n'a pas de valeur particulière. Un certain asémantisme du nom propre est ici mis en scène à travers la possibilité de commuter les termes d'un même paradigme, faisant finalement de chaque élément du paradigme un mot interchangeable : à en croire le document de Mèmes Royalistes, il serait en définitive insignifiant, puisqu'aléatoire, de s'appeler antifasciste ou libertaire. 
Néanmoins, dans le document étudié, ce n'est pas tellement l'asémantisme du nom propre en tant que signe qui est visé, mais plutôt la vacuité du vocabulaire politique et l'inanité de ses usages : dans les visuels de Mèmes Royalistes, l'asémantisme des noms de groupuscules n'est pas attribuable au nom propre comme type de nom ${ }^{8}$, mais aux usages des mots dans les discours politiques contemporains. Dit autrement, d'après le collectif royaliste, si Groupe Putschiste Européen ou Groupe Fasciste Européen n'ont pas vraiment de sens, ce n'est pas parce qu'ils sont des noms propres dotés d'une sémiotique spécifique, mais parce putschiste et fasciste sont devenus des mots vides de sens, donc interchangeables. La démarche proposée par Mèmes Royalistes, à cet égard, rappelle la vieille tradition française de critique du vocabulaire politique qui s'illustre, notamment lors de la Révolution française de 1789, par une floraison de dictionnaires polémiques qui raillent des mots vides de sens ou épinglent un lexique en perpétuel changement9.

Bien entendu, en tournant les mots en dérision en tant qu'ils seraient «vides de sens ", Mèmes Royalistes participe lui-même, à sa manière, à leur disqualification : il les présente comme des étiquettes hasardeuses, auxquelles il serait bien naïf de se fier. Quant aux conditions de production des dénominations d'organisations politiques, elles sont ramenées à des circonstances incertaines, peu dignes de crédit. Ici comme ailleurs, par l'intermédiaire de la posture réflexive sur le langage politique, les locuteurs émettent des jugements sur la vie politique elle-même. Le générateur automatique de noms de groupuscules apparaît ainsi comme un instrument de disqualification des discours politiques, mais aussi comme un outil qui contribue à produire l'effet de dévalorisation qu'il semble dénoncer.

Un regard sur une société atomisée : la syntaxe, support de représentation d'une France divisée

Comme le souligne M. Lecolle (2014), les noms propres de groupes humains constitués sont fréquemment polylexicaux. Tel fut longtemps le cas des noms de partis politiques (Parti Communiste Français, Union pour la Démocratie Française, Rassemblement Pour la République, Front National, etc.), comme permet de le retracer Maurice Tournier (1981), même si une tendance récente, qui prend acte de la désaffection des Français pour les structures politiques d'appareil, favorise la création de noms monolexicaux tels que Les Républicains ou Les Patriotes. La polylexicalité est aussi souvent de mise pour les noms de groupuscules, lesquels comportent facilement deux ou trois constituants (Unité Radicale, Faisceaux Nationalistes Européens, Brigade Antifasciste de Strasbourg).

8. Dans certains modèles de description linguistique ou logique du nom propre, celui-ci est réputé dépourvu de sens, par différence avec le nom commun. Voir des explications dans Leroy, 2004.

9. Sur ce sujet voir notamment Équipe «18 ème et Révolution », 1988. 
Dans le document étudié ici, les dénominations imaginaires sont bien entendu polylexicales : sauf à concevoir des dispositifs sans doute complexes mettant en scène des phénomènes de troncation (mots-valises, etc.) ou de composition (jeux sur les suffixes, etc.), la polylexicalité est rendue nécessaire par le principe même du générateur comme mode de création de dénominations. La caractéristique intrinsèque du générateur automatique est, en effet, de faire jouer, sur une même suite linéaire, la commutation à l'intérieur d'au moins deux paradigmes. Il convient, dès lors, d'identifier la syntaxe interne des noms polylexicaux suggérés par le document étudié, et d'en interroger les effets de sens en contexte. Notre hypothèse est que la syntaxe choisie par Mèmes Royalistes favorise une certaine représentation de la société française caractérisée par l'atomisation sociale, la division politique, et différentes sortes de fragmentation et de multiplicité.

En eux-mêmes, les titres des visuels évoquent la possibilité d'une prolifération de dénominations créatrices de groupuscules : la dimension dialogique et interpellative de l'énoncé «Toi aussi invente ton groupuscule... » (et non pas «Invente ton groupuscule... ») accroît, en discours, l'effet de démultiplication du nombre d' "actes de baptême». La "viralité » du mème (comme on dit dans le secteur du numérique), c'est-à-dire sa vocation à être diffusé par la chaîne démultipliante des recommandations des utilisateurs, accentue encore cet effet de sens.

Au plan purement quantitatif, on notera le nombre assez considérable de dénominations que permet de créer le générateur : avec les trois paradigmes correspondant aux trois constituants du syntagme, et avec des critères qui autorisent 12, 26 et 10 valeurs, le dispositif permet théoriquement la création de 5980 noms différents ("théoriquement», car le lexème front s'avère être en doublon : on aboutit donc en réalité à 2860 dénominations pour l'extrême gauche et à 3120 pour l'extrême droite). Un visuel de mème internet doit pouvoir s'afficher dans un fil d'actualité sur un smartphone. Il ne permet donc sans doute pas d'inscrire beaucoup plus de constituants et/ou de critères et/ ou de valeurs, et on peut imaginer que la possibilité, pour chaque visuel, de créer 3120 (ou 2860 ) noms propres de groupuscules, est déjà pour Mèmes Royalistes une façon de maximiser le nombre des dénominations potentielles.

La nature des constituants retient également notre attention. Nous l'avons vu, les paradigmes sont au nombre de trois. Mais, alors qu'il aurait été possible de proposer par exemple une structure en [Nom + Adjectif + Complément du nom] (qui aurait permis des dénominations telles que Division Patriote de Paris, Division Patriote de Strasbourg...), le collectif Mèmes Royalistes a choisi, à travers la structure [Nom + Adjectif + Adjectif], un système de nomination qui découpe les idées politiques de manière particulièrement fine. Le dispositif donne à voir une forte fragmentation des collectifs humains, lesquels sont appréhendés de façon morcelée quant à leurs options politiques, 
idéologiques ou doctrinales : chaque adjectif épithète vient restreindre l'extension du noyau du syntagme. Ainsi, selon le dispositif, il peut exister un groupuscule nommé Division Patriote Française, lequel se distingue d'un groupuscule Division Patriote Nationaliste, d'un groupuscule Division Patriote Identitaire... Chacun des deux adjectifs crée successivement un sous-ensemble dans l'ensemble de départ, segmentant le référent initialement visé par le nom. Bien entendu, la sémantique des unités polylexicales mériterait des commentaires plus approfondis, comme le suggèrent les analyses de M. Tournier (1981) à propos des noms de partis politiques des années 1879-1905. Mais l'essentiel est ici de pointer l'effet d'émiettement des groupuscules produit par la syntaxe. Un internaute semble avoir très bien perçu cet effet de fragmentation. Retweetant le visuel «Toi aussi invente ton groupuscule d'extrême-gauche », le titulaire du compte Pont d'Arcole l'accompagne de ce commentaire (voir annexe 2) : «Moi je viens de fonder le Parti Trotskyste Décroissant Révolutionnaire. Le PTDR... »

Tout en soulignant le caractère humoristique du propos (PTDR signifie ordinairement «pété de rire» dans les échanges numériques), le locuteur Pont d'Arcole surenchérit sur ce morcellement, tirant profit de la récursivité des langues naturelles pour ajouter un troisième adjectif au schéma syntaxique de la dénomination proposé par Mèmes Royalistes, aboutissant à une représentation plus émiettée encore.

Tous les éléments que nous venons de relever (titre dialogique, résultat élevé de la combinatoire, morcellement induit par la syntaxe) conduisent à produire symboliquement de l'atomisation et de la multiplicité. Il nous reste à indiquer quelques pistes interprétatives concernant les enjeux de cette représentation.

Un premier point concerne la vision d'une société française atomisée, découpée en autant de courants ou d'opinions que de personnes, fragmentée en autant de groupuscules que d'individus (c'est bien ce que suggèrent, symboliquement, les visuels). Certes, ce document intervient à un moment (fin 2017) où le paysage politique français est singulièrement déstabilisé dans son organisation partisane institutionnalisée, à la suite de l'élection d'Emmanuel Macron à la présidence de la République (mai 2017). Mais, au-delà, ou plutôt de manière très spécifique, le morcellement suggéré par Mèmes Royalistes parle d'autre chose, nous semble-t-il. Notre interprétation est qu'il donne crédit à la pensée maurrassienne et étaye la doctrine du nationalisme intégral. Celleci fustige l'individualisme hérité de la Révolution française, et sa croyance en un individu libre et autonome. Il pose également que l'individu est une unité qui ne doit pas primer sur les «communautés naturelles» (famille, corporations professionnelles, etc.). Quant aux opinions individuelles, le nationalisme intégral estime qu'elles doivent céder le pas à un intérêt général identifié à celui de la nation : de même que le système des partis politiques divise la France contre elle-même, la pluralité entraîne la division. En cohérence avec la pensée maur- 
rassienne, le générateur met ainsi en scène le fractionnement des opinions qui menace l'unité nationale. On pourrait nous objecter que le générateur conçu par Mèmes Royalistes a simplement des effets amusants. Mais nous soulignerons que ceux-ci ont précisément pour but de favoriser la circulation de messages émis par le collectif royaliste, et que celui-ci entend convaincre dans la durée et participer à une lutte culturelle.

Le second point concerne la question des groupuscules, et de ce que Mèmes Royalistes, comme locuteur engagé, en donne à comprendre. Comme on l'a vu, à travers le générateur proposé par Mèmes Royalistes, le «pouvoir rassembleur " (Lecolle, 2014, p. 2271) de la dénomination de groupes sociaux constitués est subverti : chaque dénomination de groupuscule ne semble en fait ici «rassembler» qu'une seule personne. Mais précisément parce que Mèmes Royalistes tourne les groupuscules en dérision, sa position est équivoque. Pour ce collectif maurrassien qui préconise le "coup de force» qui abattra la République et renversera les institutions libérales et démocratiques, ironiser sur les groupuscules est ambivalent, bien que toujours profitable à sa cause. D'un certain côté, en banalisant les groupuscules, Mèmes Royalistes donne à voir comme in offensifs et ordinaires ces petits groupes isolés, présents un peu partout, et que l'on dit pourtant potentiellement violents. Une telle banalisation peut être lue comme un encouragement fait à chacune et à chacun de créer un tel groupe : «Toi aussi invente ton groupuscule» devrait alors en quelque sorte être pris à la lettre. D'un autre côté, en banalisant les «groupuscules d'extrêmegauche » et les "groupuscules d'extrême-droite», Mèmes Royalistes les ridiculise : il discrédite les mouvements visés par les discours contemporains sur la «montée des radicalités» (black blocs, etc.). Ce faisant, le collectif maurrassien montre implicitement combien le courant royaliste occupe une place à part, irréductible aux radicalités désordonnées, violentes et dangereuses dont la scène publique offre régulièrement le témoignage.

\section{Proposer des mots comme un «stock» de marqueurs politiques:} travailler le lexique comme signe distinctif... ou le neutraliser?

Nous terminons notre analyse par ce qui apparaît, dans ce que Mèmes Royalistes donne à voir, comme la dimension saillante du discours : le lexique, qui est mis en valeur par la structure même du générateur comme document.

La réflexion sur les lexèmes ne peut pas être dissociée du fait que Mèmes Royalistes propose une paire de visuels - l'un sur l'extrême gauche, l'autre sur l'extrême droite - et non pas uniquement un visuel sur l'un ou sur l'autre : même si, de fait, chaque mème internet a pu circuler isolément sur les réseaux sociaux, tous deux ont été produits comme un ensemble et sont à interpréter comme tels. Partant de ce constat, nous estimons qu'il existe un paradoxe entre l'effet rhétorique produit par le générateur, et celui que produit le recours 
à cette paire. De son côté, le générateur suscite - comme nous l'avons expliqué plus haut - un effet rhétorique d'interchangeabilité des mots : les mots seraient substituables, échangeables, vides de sens, insignifiants. De l'autre côté, le recours à une paire de visuels engendre un effet rhétorique inverse : les mots seraient marqués et marquants, typiques, caractéristiques, significatifs. De ce contraste rhétorique surgit la représentation de deux «stocks» de mots, lesquels fonctionneraient comme autant de marqueurs caractéristiques de l'extrême gauche d'une part et de l'extrême droite d'autre part : chaque visuel permet de dégager un «réservoir » de mots supposés fonctionner comme autant de signaux typiques de l'un ou de l'autre «bord». Mèmes Royalistes semble ainsi donner crédit à la théorie dite du «fer à cheval», selon laquelle les extrêmes se rejoignent : les différences entre extrême gauche et extrême droite se voient partiellement neutralisées, dans un mouvement où « les extrêmes se touchent », comme on l'entend parfois. Le clivage extrême gauche/extrême droite se trouve déconstruit, ramené à une chimère ou à une illusion. L'analyse des lexèmes choisis dans le document pour la constitution de noms propres de groupuscules imaginaires nous confortera sur cette piste : en renvoyant dos à dos extrême gauche et extrême droite, le collectif Mèmes Royalistes se distingue de ces deux ensembles, et œuvre ainsi à la légitimation du courant maurrassien. Différentes observations vont nous conduire à étayer cette idée.

Une mise à plat des lexèmes proposés dans les visuels permet de totaliser 19 noms différents, et 66 adjectifs différents. Un premier constat porte sur les adjectifs : nous remarquons que Mèmes Royalistes a réellement maximisé le dispositif qu'il a créé, offrant une vraie diversité de vocabulaire aux utilisateurs ${ }^{10}$. En effet, on observe qu'aucun adjectif n'est commun aux paradigmes 2 et 3 (autrement dit, dans un même visuel, un adjectif déterminé est soit en position 2 soit en position 3). Ainsi, non seulement est éliminée la probabilité de voir surgir des dénominations un peu absurdes telles que Légion Solidariste Solidariste ou CollectifMarxiste Marxiste, mais, surtout, Mèmes Royalistes donne à voir à son lecteur une réelle diversité de mots fonctionnant comme marqueurs : l'effet d'un vaste «stock» de mots n'est pas artificiel.

Un deuxième constat porte sur la répartition des unités dans les visuels. En effet, on observe que quelques lexèmes sont communs aux deux visuels. Ceci concerne 4 lexèmes sur 19 pour les noms (front, action, groupe, section), et 6 lexèmes sur 66 pour les adjectifs (autonome, populaire, secret, libérateur, radical, révolutionnaire). Cette communauté de lexique peut être interprétée diversement. Une première interprétation consiste à dire que, du point de vue de Mèmes Royalistes, extrême gauche et extrême droite ne sont pas si éloignées qu'il y parait (thèse du «fer à cheval»), puisqu'elles ont des marqueurs

10. Le doublon sur le lexème nominal front apparaît comme la seule « faille » du dispositif comme instrument de maximisation des dénominations possibles. 
communs. Une seconde interprétation, non exclusive, amène à considérer que Mèmes Royalistes prend acte du fait que les mots prennent sens dans leur environnement, et qu'un même lexème produit des inflexions contrastées selon les autres lexèmes qui l'entourent : révolutionnaire n'a pas le même sens selon qu'il est cooccurrent de autogestionnaire ou de aryen. De fait, la valeur de révolutionnaire est différente dans Alternative Féministe Révolutionnaire et dans Armée Régionaliste Révolutionnaire.

Une troisième observation concerne la dimension diachronique du lexique mobilisé. Si les termes anciennement rattachés à l'extrême gauche et à l'extrême droite ne sont pas absents des visuels (mais il ne s'agit en aucun cas de termes tombés en désuétude), le document témoigne aussi d'un lexique d'apparition plus récente : anti-antifa (qui semble être apparu dans les années 1990), zadiste (apparu au début des années 2010, il s'est rapidement diffusé), insoumis (en lien avec La France insoumise, mouvement lancé par Jean-Luc Mélenchon début 2016)... Le collectif Mèmes Royalistes signale ainsi que son propos vise bel et bien la vie politique contemporaine et l'actualité du temps présent, et non pas le passé ou un monde parallèle. La dimension critique du document l'emporte ainsi en crédibilité. Dans cet exercice humoristique de réflexivité langagière, Mèmes Royalistes signale aussi sa compétence de locuteur ordinaire en matière de sentiment néologique et sa capacité à relever des mots «dans l'air du temps» (Lecolle, 2012).

Une quatrième observation nous amène à penser que la disqualification portée par Mèmes Royalistes à l'encontre de l'extrême gauche est plus forte que celle qui vise l'extrême droite. En effet, plusieurs choix lexicaux pèsent en défaveur de l'extrême gauche. On relève une surreprésentation d'adjectifs préfixés en anti- dans les adjectifs imputés à l'extrême gauche (anticapitaliste, antifasciste, antiraciste, antisioniste, mais uniquement anti-antifa à l'extrême droite), ce qui donne à voir l'extrême gauche comme particulièrement pauvre en propositions originales, et essentiellement limitée à un rôle d'opposition et de refus : l'extrême gauche serait avant tout «contre ». On relève également, pour l'extrême gauche, une surreprésentation des adjectifs formés par dérivation d'un nom propre de personne (marxiste, léniniste, trotskiste, maoïste, alors que l'extrême droite n'en comporte aucun, quand pourtant étaient théoriquement disponibles barrésien, hitlérien, mussolinien, franquiste, ou encore... maurrassien). L'extrême gauche apparaît ici dans son enfermement idéologique et doctrinaire, son incapacité à former un cadre de pensée indépendamment de la tutelle d'une autorité incarnée.

Un autre point essentiel tiré de nos observations concerne la capacité - ou plutôt l'incapacité - de l'auteur du document à s'inclure dans le mouvement humoristique qu'expriment les visuels. Si le générateur proposé tourne bel et bien en dérision l'extrême gauche et l'extrême droite, il n'est manifestement pas un support d'autodérision. On observe en effet que certains des 
lexèmes qui auraient été les plus forts marqueurs de l'auteur du document sont précisément absents : royaliste, monarchiste et maurrassien ne font pas partie des 66 adjectifs listés. Ces absences, dans une liste dont nous avons précédemment souligné l'amplitude, méritent interprétation. La nôtre est que Mèmes Royalistes signifie ainsi clairement qu'il s'exclut du clivage extrême gauche/extrême droite, par lequel il ne se sent pas concerné, et au-dessus duquel il s'élève - ou à côté duquel il se pose en alternative. Cette interprétation confirme la seconde hypothèse que nous faisions, plus haut, à propos des groupuscules et de la "montée des radicalités» : le fait de tourner en ridicule les "groupuscules d'extrême-gauche» et les "groupuscules d'extrêmedroite » est une façon, pour le collectif Mèmes Royalistes, de suggérer que le courant royaliste maurrassien occupe une place à part.

Pour finir, la place du mot fasciste interroge, et son analyse nous conforte dans le rapport très spécifique que le document étudié entretient avec la pensée maurrassienne. On observe que pas moins de 4 lexèmes empruntent à fasciste, sur un ensemble de 66 adjectifs : antifasciste à l'extrême gauche, et fasciste, néo-fasciste, anti-antifa à l'extrême droite. Cette forte présence de fasciste, sous diverses formes, semble démonétiser conjointement l'accusation de «fasciste» et la prétention à être «antifasciste». De fait, dans le magazine Création, un long article intitulé "Pour une éradication du "fascisme" dans le langage politique $»^{11}$, explique que le mot fasciste est «vide de sens », et qu'il ne correspond plus guère qu'à un stéréotype verbal, servant notamment à insulter l'adversaire. L'article se termine par un appel à se défaire de la chimérique opposition «fasciste/antifasciste », qui n'aurait pas de pertinence dans la culture politique française, et que le royalisme propose de dépasser :

Le fascisme n'est qu'un rêve chez des individus sacrifiant l'éthique à l'esthétisme. Il est temps de remettre au centre le «Politique d'abord! » et de dépasser ce faux clivage entre «fascistes» et « antifascistes».

Dans le générateur automatique de noms de groupuscules étudié, l'accent mis sur le caractère dérisoire de l'opposition entre fasciste et antifasciste est ainsi mis au service de la pensée maurrassienne. En cohérence avec les formes de banalisation que nous avons évoquées plus haut, il s'agit pour Mèmes Royalistes de disqualifier d'autres formes de radicalités que la sienne, en les taxant d'ordinaires et usées, dans un regard de dédain amusé. Outre leur caractère drolatique, qui en favorise la diffusion sur internet, les visuels conçus par Mèmes Royalistes apparaissent ainsi clairement, dans notre interprétation, comme de sérieux vecteurs de la doctrine du nationalisme intégral de Charles Maurras.

11. «Pour une éradication du “fascisme” dans le langage politique», Création, n 1 , juin 2018, p. 24-25. 


\section{En conclusion : l'apport de l'étude des noms d'organisations imaginaires à l'analyse des discours}

Parl'intermédiaire d'une posture réflexive sur le langage (commentaires métadiscursifs, jeux de mots, etc.), un locuteur émet toujours, de manière plus ou moins directe, des appréciations sur le monde, son histoire, son organisation, ses acteurs... L'étude des représentations sur la langue et sur les discours en contexte sociopolitique constitue ainsi toujours un mode d'accès à des enjeux de positionnement, à des façons pour les locuteurs de prendre place dans la conflictualité, aux manières dont ils «font avec » la division comme avec le rassemblement. Dans le cas qui nous occupe, nous avons vu combien l'expression de représentations langagières est l'occasion, pour Mèmes Royalistes, d'émettre des jugements sur la vie politique française. Nous avons suggéré la pluralité des effets de sens possibles produits par ces visuels humoristiques, en particulier dans le contexte de réseaux sociaux numériques qui favorisent la décontextualisation et des prises en charge énonciatives très différenciées. L'étude de la réflexivité langagière, du métalangage ordinaire, de différentes formes de jeux sur le langage, ici comme dans bien d'autres contextes, s'avère enrichissante, et nous espérons avoir montré ce qu'une telle analyse peut apporter à l'étude des noms propres d'organisations politiques, que celles-ci soient fictives ou plus réelles. Nous avons aussi montré combien l'étude des noms de groupes imaginaires renseigne sur le réel, pour peu qu'on prenne la peine, comme toujours en analyse du discours, de placer les phénomènes étudiés dans le contexte de leur énonciation, et de les relier aux manifestations génériques, syntaxiques et lexicales qui les rendent observables.

\section{Références}

ALBERTINI Dominique, DouCET David, 2016, La fachosphère : comment l'extrême droite remporte la bataille d'Internet, Paris, Flammarion.

Clément Arnaud, Fernandez Valérie, Puel Gilles, 2016, «Les mèmes Internet ont-ils un mode de propagation spécifique? Analyse à partir de topogrammes de mèmes de l'Internet chinois », Réseaux, nº 195, p. 107-130.

DÉzÉ Alexandre, 2011, «Un parti “virtuel” ? Le Front national au prisme de son site internet», dans Continuerlalutte.com : les partis politiques sur le web, F. Greffet éd., Paris, Presses de Sciences Po, p. 142-157.

ÉQUIPE «18 ème ET RÉVOLUTION», 1988, Dictionnaire des usages socio-politiques (17701815), fasc. 3, Dictionnaires, normes, usages, Paris, Klincksieck.

Gautier Antoine, Sıouffi Gilles éd., 2016, Les mèmes langagiers : propagation, figement, déformation [numéro thématique], Travaux de linguistique, nº 73 .

Krieg-Planque Alice, 2018, "Langue de bois», dans Publictionnaire. Dictionnaire 
encyclopédique et critique des publics, B. Fleury, M. Lecolle et J. Walter éd., Université de Lorraine, http://publictionnaire.huma-num.fr/langue-de-bois (consulté le 10 mars 2019).

- 2015, "Construire et déconstruire l'autorité en discours : le figement discursif et sa subversion », Mots. Les langages du politique, n0107, p.115-131.

LECOLLE Michelle, 2016, « Noms collectifs humains : nomination et prédication », Argumentation et analyse du discours, $\mathrm{n}^{0} 17$, http://aad.revues.org/2208 (consulté le 10 mars 2019).

- 2014, «Dénomination de groupes sociaux : approche sémantique et discursive d'une catégorie de noms propres", dans $4^{e}$ Congrès mondial de linguistique française, F. Neveu, P. Blumenthal, L. Hriba et al. éd., Les Ulis, EDP Sciences, p. 2265-2281, http://dx.doi.org/10.1051/shsconf/20140801063 (consulté le 10 mars 2019).

- 2012, "Sentiment de la langue, sentiment du discours : changement du lexique, phraséologie émergente et "air du temps" ", Diachroniques, n² 2, p.59-80.

LEROY Sarah, 2004, Le nom propre en français, Paris, Gap, Ophrys.

TOURNIER Maurice, 1981, «Vers une grammaire des dénominations sociopolitiques au début de la Troisième République (1879-1905) », Mots, n² 2, p. 51-72.

YAGUello Marina, 2006, Les langues imaginaires : mythes, utopies, fantasmes, chimères et fictions linguistiques, Paris, Seuil. 


\section{Annexes}

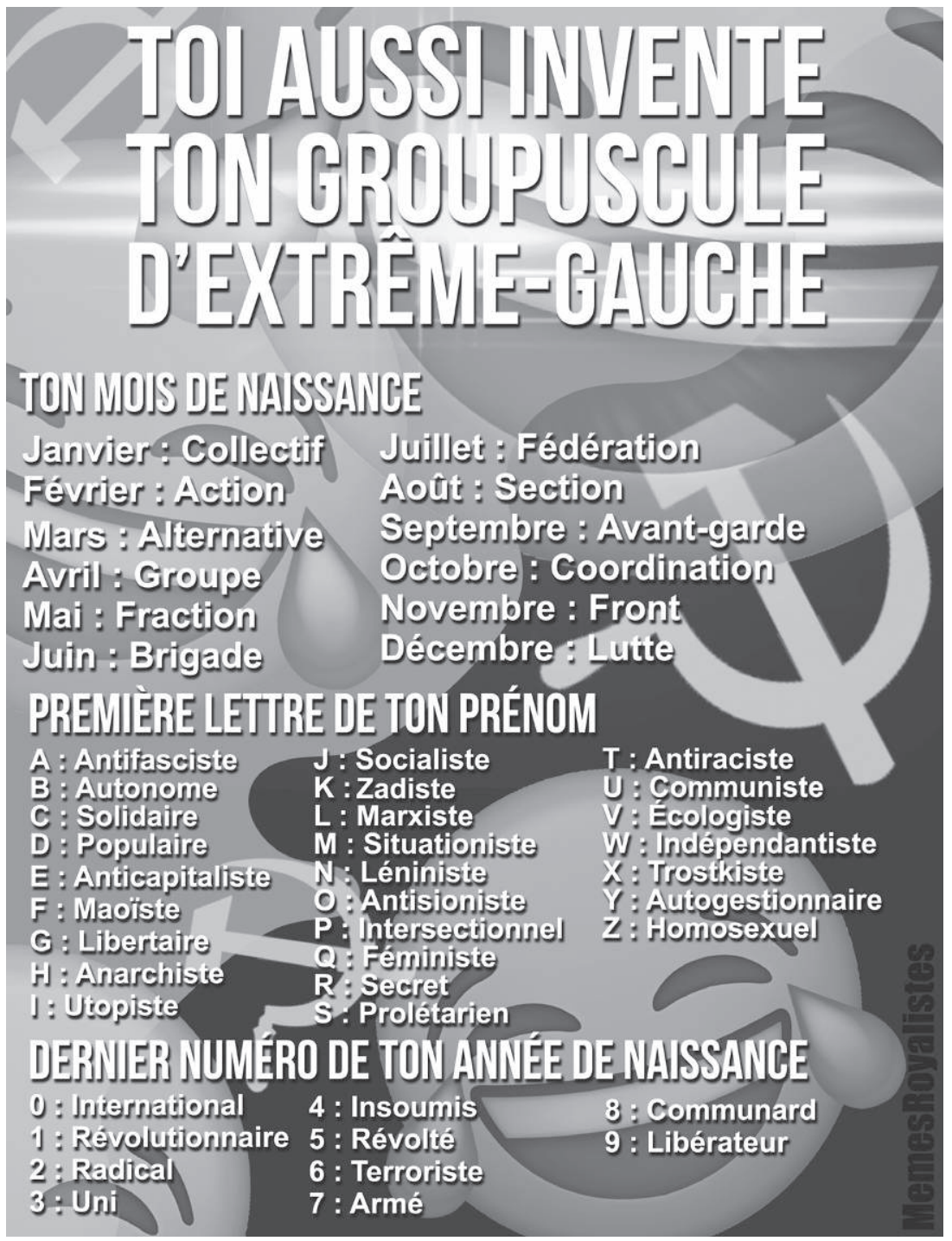

Annexe 1. "Toi aussi invente ton groupuscule d'extrême-gauche».

Visuel publié sur les comptes Facebook et Twitter de Mèmes Royalistes, 2017

https://twitter.com/MemesRoyaliste (consulté le 10 mars 2019) 


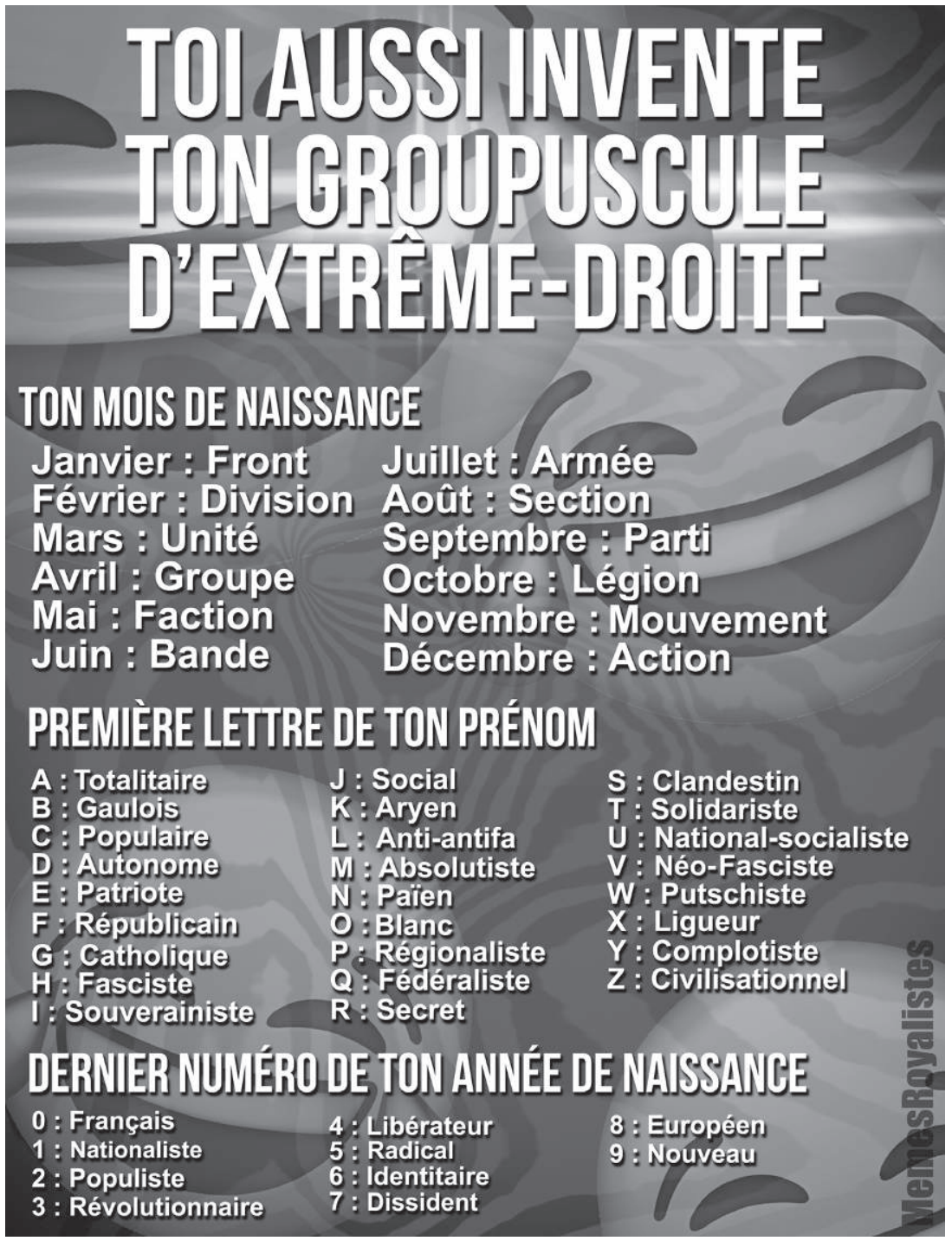

Annexe 1 bis. "Toi aussi invente ton groupuscule d'extrême-droite ".

Visuel publié sur les comptes Facebook et Twitter de Mèmes Royalistes, 2017 https://twitter.com/MemesRoyaliste (consulté le 10 mars 2019) 
Toi aussi invente ton groupuscule d'extrêmegauche ! Moi je viens de fonder le Parti Trotskyste Décroissant Révolutionnaire. Le PTDR...

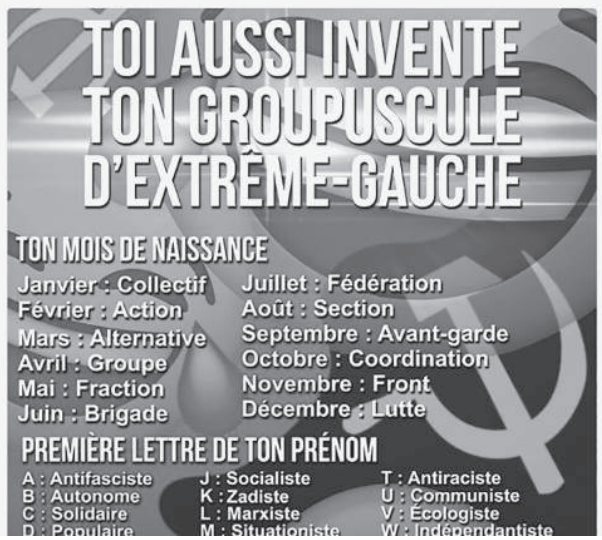

Annexe 2. Commentaire d'un document de Mèmes Royalistes par le compte Twitter de Pont d'Arcole, 2018 https://twitter.com/arcole_pont (consulté le 10 mars 2019)

\section{DIPLOME DE LEECOLE NATIONALE DADMINISTRATION, LUCIEN GRAN GARGON ENTRE TRES RAPIDE- HENT DANS LA VIE POLITIQUE EN HICITANT AU SEIN DE L'UNION DEMOCRATIQUUE DES DEMOCRATES POUR LA DEMOCRATIE (UDDD)...}

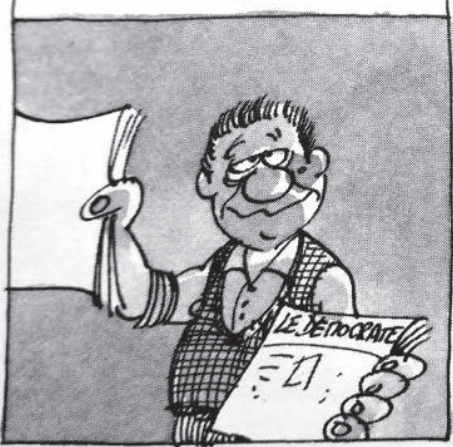

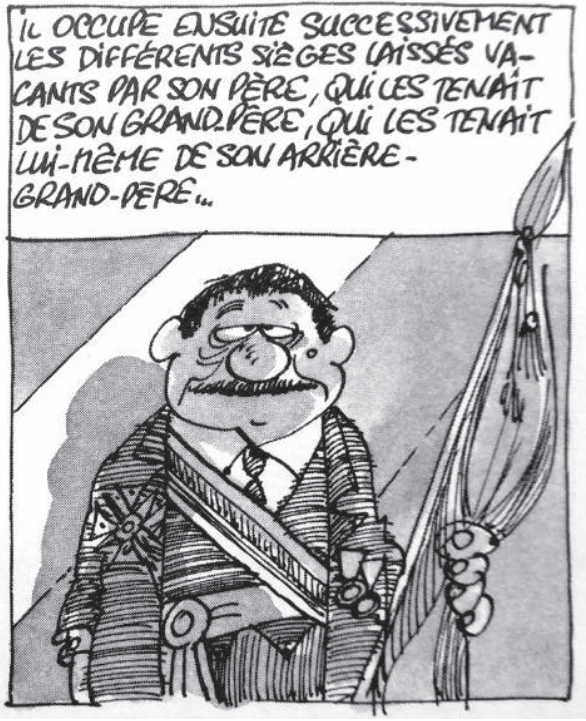

Annexe 3. Détail d'une planche de l'album :

Binet, Monsieur le Ministre : un destin national, Paris, Éditions Audie, coll. Fluide Glacial, 1989, p. 37 
Memes Islamogauchistes

12 juin $\cdot \odot$

Petit tuto pour défendre correctement NOOOTRE PROOOJEEEETTM !

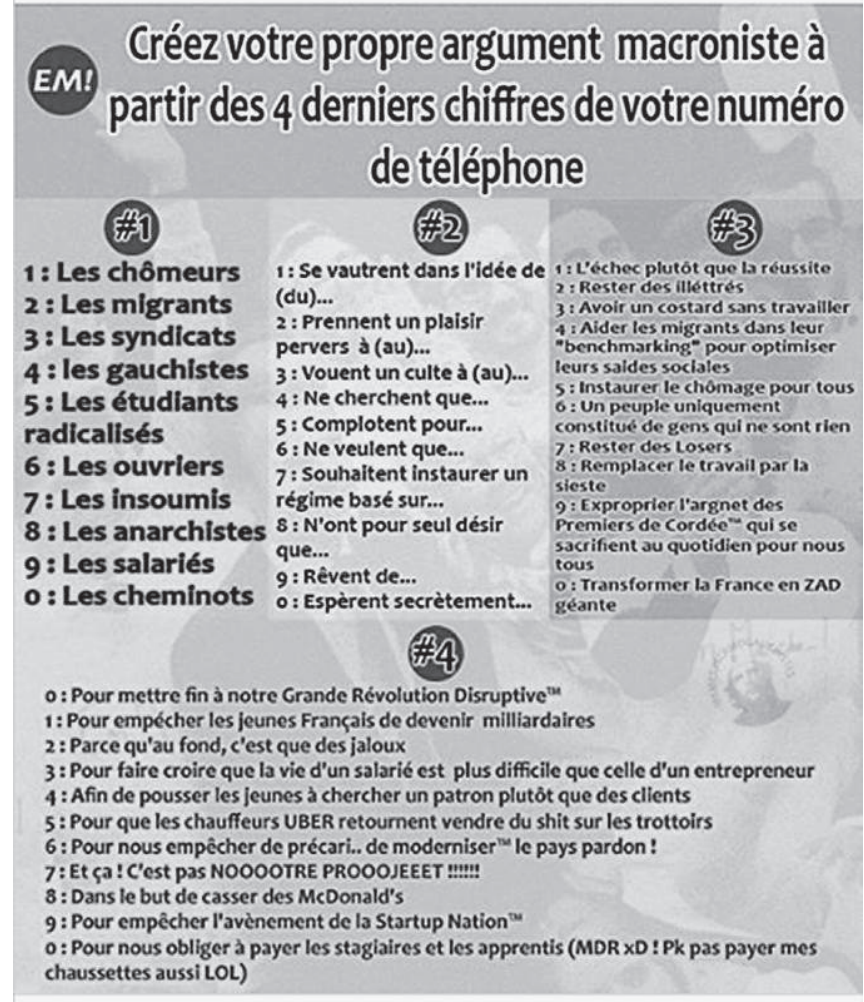

Annexe 4. Message publié sur le compte Facebook de Mèmes Islamogauchistes, 12 juin 2018 https://www.facebook.com/islamogauchisme (consulté le 10 mars 2019) 


\section{Résumé / Abstract / Compendio}

\section{Les noms d'organisations politiques imaginaires : analyse d'un générateur automatique de noms de groupuscules}

Ce travail contribue à l'étude des noms propres d'organisations politiques. Le sujet est abordé à travers un type de dénomination dont la particularité est de concerner des organisations imaginaires. Ici, le document analysé comporte deux visuels humoristiques («Toi aussi invente ton groupuscule d'extrême-gauche» et «Toi aussi invente ton groupuscule d'extrême-droite»), relevant d'un dispositif créatif appelé "générateur automatique d'énoncés». Ces visuels ont été conçus en 2017 par le collectif Mèmes Royalistes, présent sur les réseaux sociaux numériques. Nous montrons comment l'expression de représentations langagières participe, ici, de jugements sur la vie politique française. Dans le cas présent, l'ancrage royaliste et maurrassien de l'auteur du document donne à comprendre les effets de sens que nous mettons en évidence.

Mots-clés: nom propre d'organisation politique, humour, générateur automatique d'énoncés, représentations langagières, réseaux sociaux numériques

\section{About the names of imaginary political organizations: analysis of an automa- tic generator of small group names}

This work contributes to the study of the proper nouns of political organizations. The subject is addressed through a type of denomination whose particularity it is to concern imaginary organizations. Here, the document contains two humorous visuals ("Invent your own far-left group" and "Invent your own far-right group"), belonging to a creative device called "automatic statement generator". These visuals were designed in 2017 by the collective Mèmes Royalistes, which is present on social networks. We show how the expression of language representations participates here in judgments on French political life. In this case, the royalist and Maurrassian foothold of the author of the document gives us an understanding of the meanings effects that we highlight.

Keywords: political organization proper nouns, humor, automatic statement generator, linguistic representations, social networks

\section{Los nombres propios de organizaciónes políticas imaginarias: análisis de un generador automático de nombres de grupúsculos}

Este trabajo contribuye al estudio de los nombres propios de organizaciones políticas. El tema es abordado a través de un tipo de denominación cuya particularidad es concernir a organizaciones imaginarias. Aquí, el documento analizado contiene a dos visuales humorísticos («Inventa tú también tu grupúsculo de extrema-izquierda» y «Inventa tú también tu grupúsculo de extrema-derecha»), dependiendo de un dispositivo creativo llamado «generador automático de enunciados». Estos visuales han sido concebidos en 2017 por el colectivo Mèmes Royalistes, presente en las redes sociales. Mostramos cómo la expresión de representaciones lingüísticas participa, aquí, a los juicios sobre la vida política francesa. En este caso, el anclaje monárquico y maurrasiano del autor del documento da a comprender los efectos de significado que ponemos en evidencia.

Palabrasclaves: nombre propio de una organización política, humor, generador automático de enunciados, representaciones lingüísticas, redes sociales 